\title{
Resistência natural da madeira tratada de Hevea brasiliensis em ensaios de deterioração de campo
}

O objetivo desse trabalho foi avaliar a resistência natural da madeira de Hevea brasiliensis tratada com CCA, CCB e piretroide, em ensaios de deterioração de campo. Após 20 dias da aplicação dos preservantes, foi determinado o teor de extrativos totais, a massa específica básica, a porosidade e o potencial de resistência natural da madeira de Hevea brasiliensis. Os corpos de prova foram submetidos em dois ambientes: campo aberto, livre de vegetação rasteira e em floresta nativa. Periodicamente, a cada trinta dias, durante um ano, foram retirados seis corpos de prova por tratamento em cada ambiente estudado para determinação da massa seca residual. De acordo com os resultados, a madeira de Hevea brasiliensis apresentou um baixo teor de extrativos (<4\%) e um baixo potencial de resistência natural. Apenas os tratamentos preservativos realizados com CCA e CCB apresentaram diferenças estatísticas significativas, sendo que no ambiente floresta as amostras apresentaram menor massa seca residual. O índice de deterioração no ambiente floresta chegou a $70 \%$ nas madeiras não tratadas e tratadas com piretroide e a $90 \%$ nas madeiras tratadas com CCA e CCB, indicando que o tratamento com esses dois últimos preservantes é eficaz para diminuir a deterioração da madeira de Hevea brasiliensis.

\section{Natural resistance of the treated wood of Hevea brasiliensis into field test}

\begin{abstract}
The aim of this work was to evaluate the natural resistance of Hevea brasiliensis wood treated with CCA, CCB and pyrethroid in field deterioration tests. After 20 days of application of the preservatives, total extractive content, basic specific mass, porosity and natural resistance potential of Hevea brasiliensis wood were determined. The specimens were submitted to two environments: open field, free of undergrowth and native forest. Periodically, every 30 days, during one year, six specimens were removed per treatment in each environment studied to determine the residual dry mass. According to the results, the wood of Hevea brasiliensis presented a low extractive content $(<4 \%)$ and a low potential of natural resistance. Only the preservative treatments performed with $C C A$ and $C C B$ showed significant statistical differences, and in the forest environment the samples had lower residual dry mass. The deterioration index in the forest environment reached $70 \%$ in untreated and treated wood with pyrethroid and $90 \%$ in wood treated with CCA and CCB, indicating that the treatment with these last two preservatives is effective to reduce the deterioration of Hevea brasiliensis wood.
\end{abstract}

Keywords: Rubber wood; Biodeterioration; Chromated copper borate.

Topic: Ciências Florestais

Reviewed anonymously in the process of blind peer.
Received: 02/08/2020

Approved: 18/09/2020
Douglas Lamounier Faria (DD

Universidade Federal de Lavras, Brasil http://lattes.cnpq.br/2575514568061593 http://orcid.org/0000-0002-5405-8430

douglas.lamounier@yahoo.com

Tony Matheus Carvalho Eugenio (id

Universidade Federal de Lavras, Brasil

http://lattes.cnpq.br/0823437440133853

http://orcid.org/0000-0001-7049-2862

tony.matheus@hotmail.com

Thiago Moreira Cruz (D)

Universidade Federal de Lavras, Brasil http://lattes.cnpq.br/7811942414257946 http://orcid.org/0000-0003-2808-1810 thiago.moreira.cruz@gmail.com

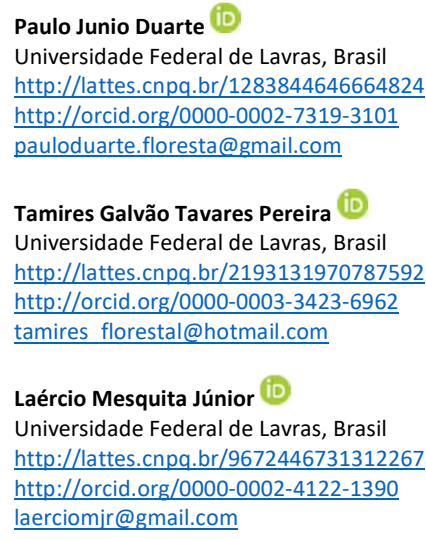
http://lattes.cnpq.br/1283844646664824 http://orcid.org/0000-0002-7319-3101 pauloduarte.floresta@gmail.com

Tamires Galvão Tavares Pereira (iD Universidade Federal de Lavras, Brasil http://lattes.cnpq.br/2193131970787592 http://orcid.org/0000-0003-3423-6962 tamires florestal@hotmail.com

Laércio Mesquita Júnior (iD Universidade Federal de Lavras, Brasil http://lattes.cnpq.br/9672446731312267 http://orcid.org/0000-0002-4122-1390 laerciomjr@gmail.com

\author{
Caik Elisio Tonelli Faria (iD \\ Universidade Federal de Lavras, Brasil \\ http://lattes.cnpq.br/0919865797988547 \\ http://orcid.org/0000-0002-0138-6801 \\ caiktonelli@gmail.com \\ Lourival Marin Mendes (iD \\ Universidade Federal de Lavras, Brasil \\ http://lattes.cnpq.br/1846557273818913 \\ http://orcid.org/0000-0001-8713-405X \\ lourivall@ufla.br \\ José Benedito Guimarães Junior (iD) \\ Universidade Federal de Lavras, Brasil \\ http://lattes.cnpq.br/5497377793349287 \\ http://orcid.org/0000-0002-9066-1069 \\ jose.guimaraes@ufla.br
}

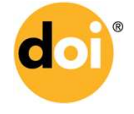

DOI: $10.6008 / C B P C 2179-6858.2020 .005 .0008$
Referencing this:

FARIA, D. L.; EUGENIO, T. M. C.; CRUZ, T. M.; DUARTE, P. J.; PEREIRA, T. G. T.; MESQUITA JÚNIOR, L.; FARIA, C. E. T.; MENDES, L. M.; GUIMARÃES JUNIOR, J. B.. Resistência natural da madeira tratada de Hevea brasiliensis em ensaios de deterioração de campo. Revista Ibero Americana de Ciências Ambientais, v.11, n.5, p.74-82, 2020. DOI: http://doi.org/10.6008/CBPC2179-6858.2020.005.0008 


\section{INTRODUÇÃO}

A madeira, por ser material de origem orgânica, dependendo das condições ambientais a que for submetida, pode ser deteriorada tanto por agentes biológicos, como micro-organismos (bactérias e fungos), insetos (coleópteros e térmitas) e brocas marinhas (moluscos e crustáceos) (BARILLARI, 2002), bem como por reações químicas, o que traz prejuízo aos seus usuários, no que se refere ao custo da mão-de-obra para substituição do material e ao risco à segurança da obra (VIVIAN, 2014).

O processo de deterioração permite analisar a vida média útil e a susceptibilidade a organismos xilófagos podendo assim ser realizado uma classificação do uso da madeira, quanto ao grau de degradação. Além disso, as informações obtidas neste tipo de estudo complementam o conhecimento das demais propriedades tecnológicas, que em conjunto, podem fornecer diretrizes para viabilizar a comercialização de espécies florestais com potencialidades na indústria madeireira (JESUS et al., 1998).

Em ambientes nos quais a madeira é suscetível à ação de organismos xilófagos, sua integridade físicomecânica tende a diminuir com o tempo de serviço em níveis que dependem de suas propriedades anatômicas e composição química (tipo e teor de extrativos e lignina), dos tratamentos preservativos caseiros (sem pressão) e industriais (com pressão) e das condições de clima (temperatura, umidade relativa e precipitação) e solo (teor de umidade, matéria orgânica e pH). Dentre esses fatores, o tratamento preservativo é o que apresenta maior facilidade de controle para prolongar a vida útil da madeira em serviço (MAGALHÃES et al., 2012).

Dentre esses tratamentos preservativos existem os tratamentos hidrossolúveis como o CCA, a base de cobre, cromo e arsênio (As); o CCB, a base de cobre, cromo e boro e os piretroides, constituído de uma solução de pentaclorofenol em solvente de petróleo, todos eficazes contra o ataque de fungos e cupins. Dentre os diversos ensaios de durabilidade, Lopez et al. (1986) consideraram os ensaios de campo um ponto fundamental na avaliação do potencial de utilização de uma espécie de madeira ou preservante.

A Hevea brasiliensis (Seringueira) vem sendo alvo de muitos estudos recentemente, devido à futura oferta de madeira dessa cultura ao final de sua rotação, uma vez que no Brasil, existem muitos povoamentos antigos. Porém pouco se sabe sobre a deterioração dessa madeira após esta passar por tratamento preservativo. Diante do exposto, o objetivo desse trabalho foi avaliar a resistência natural da madeira de Hevea brasiliensis tratada com diversos preservativos, através de ensaios de deterioração em campo.

\section{MATERIAIS E MÉTODOS}

\section{Coleta e preparação dos corpos de prova}

Três árvores de seringueira (Hevea brasiliensis), clone $\mathrm{Pb} 235$, com aproximadamente 25 anos de idade e DAP superior a $50 \mathrm{~cm}$ foram coletadas no campus da Universidade Federal de Lavras (UFLA), localizada em Lavras (altitude: 900 m; latitude: 2114'S e longitude: 4500’W) estado de Minas Gerais, Brasil. De cada árvore, toretes foram obtidos da área basal, com comprimento de 1,30 $\mathrm{m}$. Uma parte dos toretes foram tratados com CCA e CCB dentro de 24 horas após o corte das árvores. O CCB e CCA são produtos 
solúveis em água, fungicidas e inseticidas à base de borato de cobre cromatado e arseniato de cobre cromatado, respectivamente, ambos apresentando $2,5 \%$ de ingredientes ativos, possuindo boa mobilidade na madeira, sendo indicados para o tratamento de material verde.

O método de substituição de seiva foi utilizado pela transpiração radial (Figura 1). Após a fixação dos preservativos, as toras foram secas por 20 dias em temperatura ambiente e seccionadas em tábuas. Para o tratamento à base do preservativo piretroide, foi utilizado o produto Pentox. A aplicação do produto foi feita por pincelamento da madeira, após o seccionamento e obtenção das amostras para os ensaios.

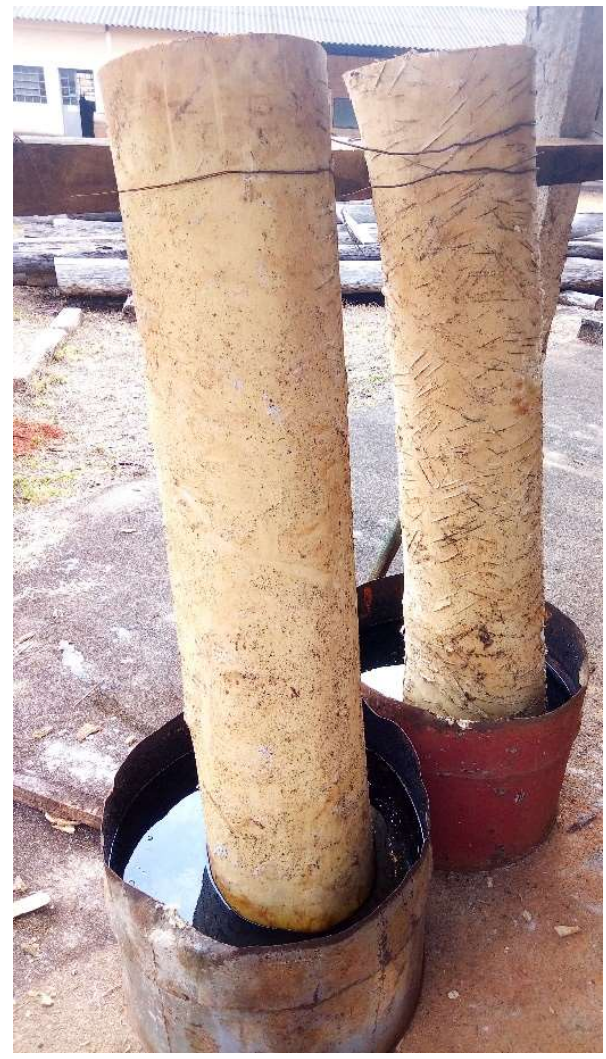

Figura 1: Toras da madeira de seringueira sendo preservadas pelo método de substituição de seiva.

Os corpos de prova para os ensaios de deterioração de campo tiveram dimensões nominais de $2,0 \times$ 2,0 × $20 \mathrm{~cm}$ (espessura, largura e comprimento, respectivamente). Logo após a confecção, os mesmos foram lixados com lixa de grã no 200 , sendo selecionados 24 corpos de prova para cada tratamento, dividindo em 2 ambientes (Tabela 1). Os corpos de prova utilizados foram apenas aqueles livres de qualquer defeito, tais como rachaduras, presença de nós e esmoado.

Tabela 1: Delineamento experimental.

\begin{tabular}{lll}
\hline \multirow{2}{*}{ Tratamentos } & Ambiente Floresta & Ambiente Campo aberto \\
\cline { 2 - 3 } & Quantidade de amostras & \\
\hline Sem tratamento & 12 & 12 \\
CCA & 12 & 12 \\
CCB & 12 & 12 \\
Piretróide & 12 & 12 \\
\hline
\end{tabular}

Antecedendo a instalação dos ensaios de deterioração, os corpos de prova foram submetidos ao condicionamento em estufa de circulação forçada de ar, com temperatura de $103{ }^{\circ} \mathrm{C}$, até atingirem massa 
constante (massa a $0 \%$ de umidade). A massa seca de cada corpo de prova foi obtida com auxílio de balança eletrônica com precisão de 0,001 g.

\section{Caracterização física e química da madeira de Hevea brasiliensis}

A partir dos corpos de prova excedentes foram preparadas amostras para determinação do teor de extrativos totais de acordo com a norma NBR 14853 (ABNT, 2010) e da massa específica básica segundo a norma NBR 11941 (ABNT, 2003). Também foram avaliadas a porosidade e o potencial de resistência natural, conforme as Equações 1 e 2, respectivamente.

$$
\Phi=1-\frac{M E b s}{M E p c} * 100
$$

Em que: $\Phi=$ porosidade (\%); MEbs= massa específica básica $\left(\mathrm{g} \cdot \mathrm{cm}^{-3}\right) ; \mathrm{MEpc}=$ massa específica da parede celular, considerando valor de $1,54 \mathrm{~g} \cdot \mathrm{cm}^{-3}$.

$$
\mathrm{Rn}=\text { MEbs } * \text { Text }
$$

Em que: $\mathrm{Rn}=$ potencial de resistência natural; MEbs= massa específica básica $\left(\mathrm{g} \cdot \mathrm{cm}^{-3}\right) ;$ Text= teor de extrativos (\%).

\section{Instalação e avaliação dos ensaios de deterioração}

A instalação dos ensaios de deterioração ocorreu em agosto de 2017, sendo realizada em dois ambientes no campus da Universidade Federal de Lavras, localizada no município de Lavras, Minas Gerais, sendo o primeiro em campo aberto (S 211' $38^{\prime \prime}$ e W 44ำ5'14"), livre de vegetação rasteira, e o segundo em floresta nativa (S 2113'41" e W 4458'07"), em área de proteção permanente.

A distribuição dos corpos de prova em cada ambiente foi definida pela divisão em quatro blocos casualizados, onde foram distribuídos doze corpos de prova aleatórios dos quatro tratamentos estudados em cada bloco (cada qual com seis linhas). Os blocos e as suas linhas foram distanciados 50 e $25 \mathrm{~cm}$ entre si, respectivamente, enquanto os corpos de prova foram distanciados $10 \mathrm{~cm}$ uns dos outros. De modo a proporcionar a exposição tanto do intemperismo biótico quanto abiótico, os corpos de prova foram soterrados até metade de seu comprimento, ou seja, $10 \mathrm{~cm}$.

Periodicamente, a cada trinta dias, durante um ano, foram retirados seis corpos de prova por tratamento em cada ambiente estudado. Após a remoção, os corpos de prova foram limpos com auxílio de uma escova para retirada do solo aderido e então submetidos ao condicionamento em estufa de circulação forçada de ar a $103{ }^{\circ} \mathrm{C}$ até obtenção de massa constante. A determinação da massa seca residual de cada corpo de prova foi realizada por meio da diferença entre as massas inicial e final (Equação 3).

$$
M S R=100-\left[\left(\frac{M i-M f}{M i}\right) \times 100\right]
$$

Em que: $M S R=$ massa seca residual, em \%; $M i=$ massa inicial, em g; $M f=$ massa final, em $g$. Por sua vez, as avaliações visuais foram procedidas ao empregar um sistema subjetivo de notas como descrito por Lepage (1970), o qual relaciona o estado de sanidade da madeira com um índice de deterioração 
(Tabela 2).

Tabela 2: Classificação do índice de deterioração da madeira.

\begin{tabular}{lll}
\hline Estado de sanidade & Nota & Índice de deterioração \\
\hline Sadio, nenhum ataque & 0 & 100 \\
Ataque leve ou superficial de fungos e térmitas & 1 & 90 \\
Ataque evidente, porém moderado de fungos e térmitas & 2 & 70 \\
Apodrecimento intenso ou ataque intenso de térmitas & 3 & 40 \\
Quebra, perda quase total de resistência & 4 & 0 \\
\hline
\end{tabular}

\section{Análise estatística}

Os resultados foram submetidos à análise estatística por meio de ANOVA. Rejeitada a hipótese de nulidade, foi aplicada a comparação de médias por meio do teste de Tukey. Todos os testes foram efetuados no programa Sisvar 5.6 a 95\% de probabilidade.

\section{RESULTADOS E DISCUSSÃO}

Os valores médios de massa específica básica, teor de extrativos, porosidade e potencial de resistência natural da madeira de Hevea brasiliensis sob os diferentes tratamentos preservativos avaliados estão apresentados na Tabela 3 a seguir.

Tabela 3: Caracterização física e química da madeira tratada de Hevea brasiliensis.

\begin{tabular}{lllll}
\hline Tratamentos & MEbs $\left(\mathrm{g} \cdot \mathrm{cm}^{-3}\right)$ & $\Phi(\%)$ & Ext (\%) & $\mathrm{Rn}$ \\
\hline Sem tratamento & $0,559 \mathrm{~A}$ & $63,70 \mathrm{~A}$ & $3,16 \mathrm{~A}$ & $1,76 \mathrm{~A}$ \\
CCA & $0,556 \mathrm{~A}$ & $63,89 \mathrm{~A}$ & $3,61 \mathrm{~B}$ & $2,01 \mathrm{~B}$ \\
CCB & $0,557 \mathrm{~A}$ & $63,83 \mathrm{~A}$ & $3,54 \mathrm{~B}$ & $1,97 \mathrm{~B}$ \\
Piretróide & $0,551 \mathrm{~A}$ & $64,22 \mathrm{~A}$ & $3,22 \mathrm{~A}$ & $1,77 \mathrm{~A}$ \\
\hline
\end{tabular}

Em que: MEbs = massa específica básica; $\Phi=$ porosidade; Ext = teor de extrativos; $\mathrm{Rn}=$ potencial de resistência natural. Médias seguidas de mesma letra, na coluna, não diferem estatisticamente entre si, pelo teste de Tukey a $5 \%$ de significância.

Segundo Panshin et al. (1980), a propriedade física massa específica e consequentemente as porosidades podem ser utilizadas como indicativo da durabilidade natural da madeira. Madeiras menos densas e mais porosas oferecem menor estabilidade à deterioração. A Hevea brasiliensis é uma espécie originária da região Amazônica (LIMA et al., 2000), e de modo geral, espécies dessa região com teor de extrativos acima de $7 \%$ apresentam um elevado potencial de durabilidade natural, e consequentemente, as perdas de massa são mínimas (CARNEIRO et al., 2009). No entanto, observa-se que para todos os tratamentos estudados o teor de extrativos ficou abaixo de 7\%, resultando em valores de potencial de resistência natural baixos. Romanini et al. (2014) também observaram valores de potencial de resistência natural baixos para outras espécies Amazônicas, encontrando 1,57 para Amescla; 2,18 para Cambará e 6,57 para Itaúba; já para Cumaru, os autores encontraram valores médios de resistência natural de 10,81 , resultado da maior massa específica básica da espécie $\left(0,94 \mathrm{~g} \cdot \mathrm{cm}^{-3}\right)$ e do maior teor de extrativos $(11,50 \%)$. Apesar da relação direta entre o teor de extrativos e a massa específica da madeira (KOLLMANN, 1959), o potencial de resistência natural, conforme descrito por Carneiro et al. (2009), não pode ser associado apenas aos dois parâmetros, mas sim com as classes químicas dos extrativos que atribuem toxidez aos organismos xilófagos (PAES et al., 
2007).

Na Tabela 4 a seguir são apresentados os valores obtidos para massa seca residual para os diferentes tratamentos preservativos, nos dois ambientes avaliados.

Tabela 4: Massa seca residual (MSR) dos quatro tratamentos da madeira de Hevea brasiliensis.

\begin{tabular}{lll}
\hline Tratamento & MSR (\%) - Floresta & MSR (\%) - Campo aberto \\
\hline Sem tratamento & $70,30 \mathrm{Aa}$ & $74,12 \mathrm{Ab}$ \\
Piretróide & $72,67 \mathrm{Aa}$ & $75,55 \mathrm{Ab}$ \\
CCB & $75,04 \mathrm{Ba}$ & $79,96 \mathrm{Bb}$ \\
CCA & $74,84 \mathrm{Ba}$ & $79,93 \mathrm{Bb}$ \\
\hline
\end{tabular}

*Médias seguidas de mesma letra, na coluna e na linha, não diferem estatisticamente entre si, pelo teste de Tukey a 5\% de significância.

Diante dos resultados apresentados acima, pode-se observar que houve diferença significativa para os tratamentos nos ambientes de exposição floresta e campo aberto. Comparando os resultados entre os dois blocos avaliados (campo aberto e floresta), observa-se que os tratamentos preservativos realizados com CCB e CCA apresentaram diferenças estatísticas significativas, resultando em maiores valores de massa seca residual (MSR).

Segundo Melo et al. (2010), maior perda de massa na floresta pode ter ocorrido em razão da alta umidade existente no ambiente de floresta, ao armazenamento de água no solo (permanecia úmido por vários dias após as chuvas) e a pouca infiltração de raios solares pelas copas, o que proporciona baixa luminosidade e temperaturas inferiores as alcançadas no ambiente de campo.

Vários autores, dentre eles Mendes et al. (1988) e Santini (1988), afirmam que o cerne é mais resistente ao ataque de organismos xilófagos que o alburno. Isso ocorre por que, ao perder a função fisiológica, uma grande quantidade de extrativos é depositada nas células do cerne. Esses extrativos podem ser tóxicos aos organismos xilófagos e, no caso específico dos fungos, dificultam o desenvolvimento das hifas e a ação de enzimas decompositoras (PAES et al., 2007).

Segundo Servolo Filho (2013), a seringueira tem como característica não apresentar diferenciação entre cerne e alburno, bem como a ausência de extrativos presentes na forma de terpenos, e os polifenóis condensados como quinonas, lignanas, taninos e estilbenos na madeira, logo, há uma maior susceptibilidade ao ataque de xilófagos.

Vários pesquisadores têm estudado a durabilidade natural em pesquisas de campo para diversas espécies de madeira, como Romanini et al. (2014) que analisaram a durabilidade natural das espécies Trattinnickia rhoifolia (amescla), Qualea albiflora (cambará), Dipteryx odorata (cumaru) e Mezilaurus itauba (itaúba), verificando teores de massa seca residual acima de 95\% para cambará, cumaru e itaúba, já para amescla os valores médios foram de 76,30\%, próximos aos observados neste trabalho. Vale ressaltar que os maiores teores de massa seca residual encontrados pelos autores são devido aos maiores valores médios de massa específica básica e maiores teores de extrativos. A ação inseticida e fungicida dos tratamentos preservativos realizados também foi verificada por Araújo et al. (2012), cujos autores trabalhando com a madeira de eucalipto citriodora (Corymbia citriodora (Hook.) K.D. Hill \& L.A.S. Johnson) tratada com CCA por processo a vácuo e pressão, observaram menores teores de biodeterioração para a madeira tratada. Os 
resultados obtidos neste trabalho mostram que a madeira preservada com os preservativos hidrossolúveis tipos CCA e CCB apresentaram maiores teores de massa seca residual em relação à madeira sem tratamento, mesmo que aplicados por meio de processos sem pressão.

Em relação aos dois ambientes de exposição analisados, observa-se que houve diferença estatística significativa entre os tratamentos, em que os corpos de prova tratados quimicamente com CCA e CCB apresentaram os maiores valores médios de massa seca residual para o ambiente campo aberto. Observa-se também que os tratamentos instalados no ambiente de campo aberto perderam menos massa para aqueles avaliados no ambiente da floresta. Esse mesmo comportamento também foi observado por Ribeiro et al. (2014), cujos autores avaliaram a durabilidade natural da madeira de jequitibá em ensaios de deterioração em campo aberto e floresta, verificando que o ambiente da floresta apresentou características edáficas mais propícias à deterioração da madeira de jequitibá.

O comportamento ao longo dos 12 meses de avaliação dos corpos de prova submetidos aos ambientes de exposição floresta e campo aberto são verificados nas Figuras 2 e 3.

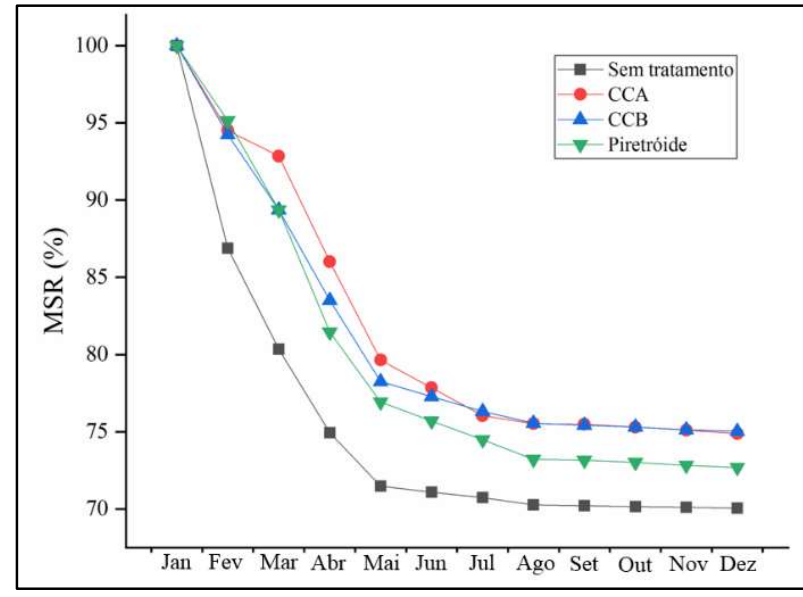

Figura 2: Teor de massa seca residual (MSR) ao longo dos 12 meses de avaliação para os corpos de prova submetidos ao ambiente floresta.

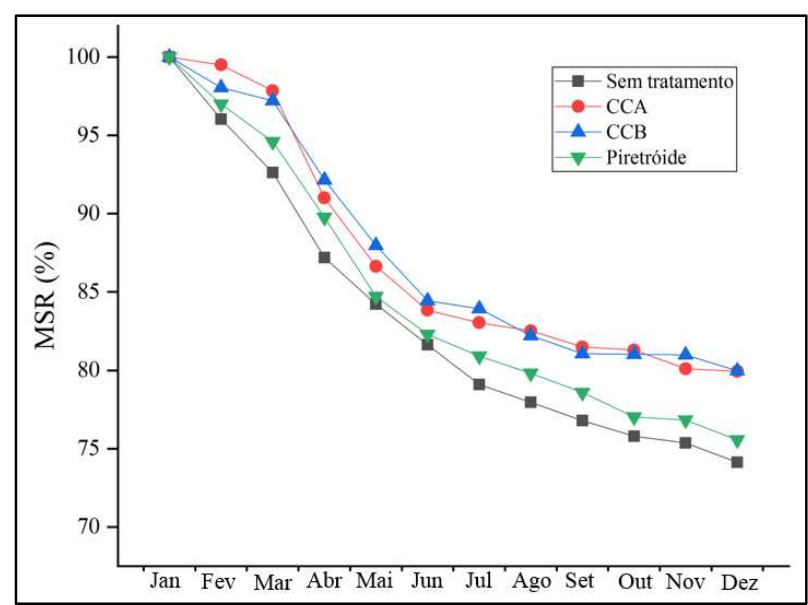

Figura 3: Teor de massa seca residual (MSR) ao longo dos 12 meses de avaliação para os corpos de prova submetidos ao ambiente campo aberto.

Observando as Figuras 2 e 3 apresentadas acima, nota-se que os corpos de prova sem tratamento preservativo submetidos aos ambientes floresta e campo aberto apresentaram uma elevada perda de massa nos primeiros 5 meses, logo em seguida houve estabilização da perda de massa ao longo dos próximos meses. Comportamento reverso foi observado para os corpos de prova preservados quimicamente, onde nota-se que a perda de massa ao longo dos meses não foi tão acentuada. Apesar de ocorrida perda de massa para os corpos de prova preservados com CCA e CCB, ambos tratamentos diferiram estatisticamente dos demais (sem tratamento e tratado com piretroide), como observado na Tabela 3.

A maior perda de massa nos dois ambientes avaliados nos 5 primeiros meses pode ser atribuída às condições climáticas, onde que no período de outubro a março há uma maior precipitação média (EMBRAPA, 2010). Já ao longo de todo período de análise, a alta perda de massa ocorrida para a Hevea brasiliensis pode ser justificada devido seu caráter próprio ao apresentar alto teor de carboidratos no alburno de sua madeira, de aproximadamente $8 \%$, na forma de grãos de amido distribuídos no interior das células de seu parênquima, 
quando comparadas a madeiras de outras espécies que oscilam entre 1,0 a 3,0\% (SANTANA et al., 1999), valores confirmados por Milingliang et al. (2008) onde os autores constataram altos teores de amido (7,5 a 10,2\%) e açúcares livres (1,0 a 2,3\%).

Na Tabela 5 a seguir é apresentado os índices de deterioração dos diferentes tratamentos preservativos para as amostras de Hevea brasiliensis, nos dois ambientes avaliados:

Tabela 5: Comparações múltiplas do índice de deterioração (\%) para a interação entre ambiente x período.

\begin{tabular}{lll}
\hline \multirow{2}{*}{ Tratamento } & \multicolumn{1}{l}{ Índice de deterioração (\%) } \\
\cline { 2 - 3 } & Ambiente & Campo Aberto \\
\cline { 2 - 3 } & Floresta & $90,00 \mathrm{Ab}$ \\
Sem tratamento & $70,00 \mathrm{Aa}$ & $90,00 \mathrm{Ab}$ \\
Piretróide & $70,00 \mathrm{Aa}$ & $90,00 \mathrm{Aa}$ \\
CCB & $90,00 \mathrm{Ba}$ & $90,00 \mathrm{Aa}$ \\
CCA & $90,00 \mathrm{Ba}$ & \\
\hline
\end{tabular}

*Médias seguidas de mesma letra, na coluna e na linha, não diferem estatisticamente entre si, pelo teste de Scott-Knott a $5 \%$ de significância.

Na Tabela 5 acima pode-se observar que houve diferença estatística significativa tanto entre o ambiente de exposição das amostras quanto nas amostras expostas ao ambiente da floresta estabelecida. Apenas não houve diferença estatística entre os tratamentos expostos em campo aberto. Comparando os resultados de índice de deterioração com àqueles de massa seca residual, verifica-se que os resultados observados para massa seca residual e índice de deterioração apresentaram resultados distintos para os dois ambientes de exposição. O mesmo comportamento foi observado por Melo et al. (2010), que verificaram diferenças no índice de deterioração apenas. Ainda segundo os autores, esses resultados evidenciam que a subjetividade do índice de deterioração, avaliado por meio de notas, pode subestimar ou superestimar a resistência do material testado. Apesar disso, os autores observaram boa correlação entre a perda de massa e o índice de deterioração. Romanini et al. (2014) observaram a mesma tendência entre massa seca residual e índice de deterioração, onde as espécies com maiores valores médios de massa seca residual apresentaram os maiores índices de deterioração.

\section{CONCLUSÕES}

Pode-se concluir que apenas a madeira de seringueira sem tratamento preservativo e tratada com piretroide nos ambientes floresta e campo aberto apresentaram menor massa seca residual, sendo estas de 70,30 e $72,67 \%$, respectivamente.

Os baixos teores de massa seca residuais podem ser atribuídos às propriedades inerentes da madeira de seringueira, uma vez que esta espécie não apresenta distinção entre cerne e alburno, bem como pelos baixos teores de extrativos, principalmente ausência de alguns grupos de extrativos, que agem como barreira para entrada de agentes xilófagos.

O índice de deterioração no ambiente da floresta chegou a 70\% nas madeiras não tratadas e tratadas com piretroide e a $90 \%$ nas madeiras tratadas com CCA e CCB, indicando que o tratamento com esses dois últimos preservantes é eficaz para diminuir a deterioração da madeira de seringueira. 


\section{REFERÊNCIAS}

ARAÚJO, H. J. B.; MAGALHÃES, W. L. E.; OLIVEIRA, L. C.. Durabilidade de madeira de eucalipto citriodora (Corymbia citriodora (Hook.) K.D. Hill \& L.A.S. Johnson) tratada com CCA em ambiente amazônico. Acta Amazônica, Manaus, v.42, n.1, p.49-58, 2012.

ABNT. Associação Brasileira de Normas Técnicas. NBR 11941: madeira: determinação da densidade básica. Rio de Janeiro: ABNT, 2003.

ABNT. Associação Brasileira de Normas Técnicas. NBR 14853: Madeira: Determinação do material solúvel em etanol-tolueno e em diclorometano e em acetona. Rio de Janeiro: ABNT, 2010.

BARILLARI, C. T.. Durabilidade da madeira do gênero Pinus tratada com preservantes: avaliação em campo de apodrecimento. Tese (Doutorado) - Universidade de São Paulo, São Paulo, 2002.

CARNEIRO, J. S.; EMMERT, L.; STERNADT, G. H.; MENDES, J. C.; ALMEIDA, G. F.. Decay susceptibility of Amazon wood species from Brazil against white rot and brown rot decay fungi. Holzforschung, Berlin, v.63, n.6, p.767- 772, 2009.

EMBRAPA. Empresa Brasileira de Pesquisa Agropecuária. Boletim de Pesquisa e Desenvolvimento. Índices Pluviométricos em Minas Gerais. EMBRAPA, 2010.

JESUS, M. A.; MORAES, J. W.; CARDIAS, M. F. C.; ABREU, R. L. S.. Durabilidade natural de 46 espécies de madeira Amazônica em contato com o solo em ambiente florestal. Scientia Forestalis, Piracicaba, v.54, p.81-92, 1998.

KOLLMANN, F.. Tecnologia de la madeira y sus applicaciones. Madrid: Reunidas S.A., 1959.

LEPAGE, E. S.. Método sugerido pela IUFRO para ensaios de campo com estacas de madeira. Preservação de madeiras, São Paulo, v.1, n.4, p.205-216, 1970.

LIMA, R. R.; TOURINHO, M. M.; COSTA, J. P. C.. Várzeas fluvio-marinhas da Amazônia brasileira: características e possibilidades agropecuárias. Belém: FCAP, 2000.

LOPEZ, G. A. C.; MILANO. S.. Avaliação da durabilidade natural da madeira e de produtos usados na sua proteção. In: LEPAGE, E. S.. Manual de preservação de madeiras. São Paulo: IPT, 1986. p.473-510.

MAGALHÃES, W. L. E.; MATTOS, B. D.; MISSIO, A. L.. Field testing of CCA-treated Brazilian spotted gum. International Biodeterioration \& Biodegradation, v.74, p.124-128, 2012.
MELO, R. R.; STANGERLIN, D. M.; SANTINI, E. J.; HASELEIN, C. R.; GATTO, D. A.; SUSIN, F.. Durabilidade natural da madeira de três espécies florestais em ensaios de campo. Ciência Florestal, Santa Maria, v.20, n.2, p.357-365, 2010.

MENDES, A. S.; ALVES, M. V. S.. A degradação da madeira e sua preservação. Brasília: IBDF, 1988.

MILINGLIANG, J.; ZHIJUAN, W.. Rubberwood preservation by friendly preservatives. In: Promotion of rubberwood processing technology in the Asia-Pacific Region. Haikon: ITTO, 2008. p.177-128.

PAES, J. B.; MELO, R. R.; LIMA, C. R.. Resistência natural de sete madeiras a fungos e cupins xilófagos em condições de laboratório. Cerne, Larvas, v.13, n.2, p.160-169, 2007.

PANSHIN, A. J.; ZEEUW, C.. Text book of wood technology. 4. ed. New York: Mc Graw Hill, 1980.

RIBEIRO, M. A.; STANGERLIN, D. M.; SOUZA, A. P.; CARDOSO, G. V.; CALEGARI, L.; GATTO, D. A.. Natural durability of the jequitibá wood in deterioration tests in open field and forest during the dry and rainy seasons. Comunicata Scientiae, Bom Jesus, v.5, n.4, p.402-411, 2014.

ROMANINI, A.; STANGERLIN, D. M.; PARIZ, E.; SOUZA, A. P.; GATTO, D. A.; CALEGARI, L.. Durabilidade natural da madeira de quarto espécies amazônicas em ensaios de deterioração de campo. Nativa, Sinop, v.02, n.01, p.13-17, 2014.

SANTANA, M. A. E.; EIRAS, K. M. M.. Madeira de Hevea brasiliensis: adequação tecnológica para sua utilização. Brasília: IBAMA, 1999.

SANTINI, E. J.. Biodeterioração e preservação da madeira. Santa Maria: CEPEF, 1988.

SERVOLO FILHO, H. J.. Propriedades mecânicas da madeira de clones de seringueira (Hevea brasiliensis-RRIM600 E GT1) analisadas em duas épocas do seu ciclo fenológico anual. Tese (Doutorado em Recursos Florestais) Universidade de São Paulo, São Paulo, 2013.

VIVIAN, M. A.; SANTINI, E. J.; MODES, K. S.; CARVALHO, D. E.; MORAIS, W. W. C.. Resistência biológica da madeira tratada de duas espécies de Eucalyptus em ensaio de campo. Pesquisa Florestal Brasileira, v.34, n.80, p.425-433, 2014.

A CBPC - Companhia Brasileira de Produção Científica (CNPJ: 11.221.422/0001-03) detém os direitos materiais desta publicação. Os direitos referem-se à publicação do trabalho em qualquer parte do mundo, incluindo os direitos às renovações, expansões e disseminações da contribuição, bem como outros direitos subsidiários. Todos os trabalhos publicados eletronicamente poderão posteriormente ser publicados em coletâneas impressas sob coordenação da Sustenere Publishing, da Companhia Brasileira de Produção Científica e seus parceiros autorizados. Os (as) autores (as) preservam os direitos autorais, mas não têm permissão para a publicação da contribuição em outro meio, impresso ou digital, em português ou em tradução. 